From the University of California, San Francisco, Comprehensive Cancer Center, San Francisco, CA.

Submitted July 9, 2008; accepted October 9, 2008; published online ahead of print at www.jco.org on December 15, 2008

Supported by National Cancer Institute Specialized Program of Research Excellence Grant No. 2 P50 CA097257 and Genentech Inc.

This report describes original work performed at the University of California, San Francisco, Comprehensive Cancer Center.

Authors' disclosures of potential conflicts of interest and author contributions are found at the end of this article.

Clinical Trials repository link available on JCO.org.

Corresponding author: Michael Prados, MD, University of California, San Francisco, 400 Parnassus Ave, Rm A808, San Francisco, CA 94143; e-mail: pradosm@neurosurg.ucsf.edu.

(C) 2008 by American Society of Clinical Oncology

0732-183X/09/2704-579/\$20.00

DOI: 10.1200/JCO.2008.18.9639

\title{
Phase II Study of Erlotinib Plus Temozolomide During and After Radiation Therapy in Patients With Newly Diagnosed Glioblastoma Multiforme or Gliosarcoma
}

Michael D. Prados, Susan M. Chang, Nicholas Butowski, Rebecca DeBoer, Rupa Parvataneni, Hannah Carliner, Paul Kabuubi, Jennifer Ayers-Ringler, Jane Rabbitt, Margaretta Page, Anne Fedoroff, Penny K. Sneed, Mitchel S. Berger, Michael W. McDermott, Andrew T. Parsa, Scott Vandenberg, C. David James, Kathleen R. Lamborn, David Stokoe, and Daphne A. Haas-Kogan

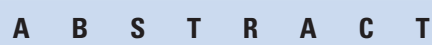

\section{Purpose}

This open-label, prospective, single-arm, phase II study combined erlotinib with radiation therapy (XRT) and temozolomide to treat glioblastoma multiforme (GBM) and gliosarcoma. The objectives were to determine efficacy of this treatment as measured by survival and to explore the relationship between molecular markers and treatment response.

\section{Patients and Methods}

Sixty-five eligible adults with newly diagnosed GBM or gliosarcoma were enrolled. We intended to treat patients not currently treated with enzyme-inducing antiepileptic drugs (EIAEDs) with 100 $\mathrm{mg} / \mathrm{d}$ of erlotinib during XRT and $150 \mathrm{mg} / \mathrm{d}$ after XRT. Patients receiving EIAEDs were to receive $200 \mathrm{mg} / \mathrm{d}$ of erlotinib during XRT and $300 \mathrm{mg} / \mathrm{d}$ after XRT. After XRT, the erlotinib dose was escalated until patients developed tolerable grade 2 rash or until the maximum allowed dose was reached. All patients received temozolomide during and after XRT. Molecular markers of epidermal growth factor receptor (EGFR), EGFRvIII, phosphatase and tensin homolog (PTEN), and methylation status of the promotor region of the MGMT gene were analyzed from tumor tissue. Survival was compared with outcomes from two historical phase II trials.

\section{Results}

Median survival was 19.3 months in the current study and 14.1 months in the combined historical control studies, with a hazard ratio for survival (treated/control) of $0.64(95 \% \mathrm{Cl}, 0.45$ to 0.91 ). Treatment was well tolerated. There was a strong positive correlation between MGMT promotor methylation and survival, as well as an association between MGMT promotor-methylated tumors and PTEN positivity shown by immunohistochemistry with improved survival.

\section{Conclusion}

Patients treated with the combination of erlotinib and temozolomide during and following radiotherapy had better survival than historical controls. Additional studies are warranted.

\section{J Clin Oncol 27:579-584. (c) 2008 by American Society of Clinical Oncology}

\section{INTRODUCTION}

Malignant gliomas are the second-leading cause of cancer mortality in people younger than 35 years and the fourth-leading cause in those younger than 54 years. ${ }^{1}$ Current management includes surgery followed by radiotherapy to $60 \mathrm{~Gy}$, with temozolomide (Temodar; Schering-Plough, Kenilworth, NJ) as an adjuvant to radiotherapy. ${ }^{2}$ Despite this regimen, patients with glioblastoma multiforme (GBM) have a median survival of approximately 14 months. A mechanism-based strategy to reduce uncontrolled cell growth is one of many approaches to cancer therapy, including for malignant glioma.
An important pathway for newly diagnosed GBM involves the epidermal growth factor receptor (EGFR). ${ }^{3}$ Amplification of EGFR is a common genetic feature of GBM and is reported in approximately $40 \%$ of cases. Approximately $75 \%$ of cases of EGFR gene amplification are associated with gene deletions and rearrangement, most commonly, deletion of the extracellular ligandbinding domain, referred to as EGFRvIII. ${ }^{4}$ This deletion leads to ligand-independent constitutive activation of the tyrosine kinase. Dysregulated EGFR signaling promotes cell proliferation, migration, invasiveness, and impaired apoptosis. ${ }^{5}$ These observations make GBM a good target for 
EGFR tyrosine kinase inhibitors such as erlotinib (OSI-774). Erlotinib inhibits human EGFR tyrosine kinase with concentration that inhibits $50 \%$ of $2 \mathrm{nmol} / \mathrm{L}(0.786 \mathrm{ng} / \mathrm{mL})$ in an in vitro enzyme assay and 20 $\mathrm{nmol} / \mathrm{L}(7.86 \mathrm{ng} / \mathrm{mL})$ in intact tumor cells. ${ }^{6}$ This inhibition is selective, results in cell-cycle arrest at $\mathrm{G}_{1}$, and is reversible. Oral administration of erlotinib in mice with human glioma xenografts reduces EGFR autophosphorylation by $70 \%$. Erlotinib and its active metabolite (OSI-420) are metabolized by the cytochromes CYP1A2, 3A4, 3A5, and $1 \mathrm{~A} 1$, and dosing in humans must be modified to account for this.

We previously reported on a phase I trial of erlotinib alone or in combination with temozolomide that showed the combination was well-tolerated, and the results of that study formed the basis for dosing schedules tested in the current study. ${ }^{7}$ The results also suggested that amplification of EGFR and presence of intact phosphatase and tensin homolog (PTEN) are correlated with response. ${ }^{8}$ After the phase I trial, we designed an open-label, single-arm, phase II trial that adds erlotinib to the standard therapy of radiation and temozolomide for treating GBM and gliosarcoma (GS).

\section{PATIENTS AND METHODS}

\section{Patient Population}

Eligibility criteria for this protocol included the following: $\geq 18$ years of age, Karnofsky Performance Score (KPS) $\geq 60$, and newly diagnosed intracranial GBM or GS confirmed by biopsy or resection no more than 5 weeks before treatment. No prior treatment was allowed. Patients needed adequate bone marrow function $(\mathrm{WBC} \geq 3,000 / \mu \mathrm{L}$, absolute neutrophil count $\geq 1,500 / \mu \mathrm{L}$, platelet count $\geq 100,000 / \mu \mathrm{L}$, and hemoglobin $\geq 10$ $\mathrm{gm} / \mathrm{dL}$ ), liver function (AST and bilirubin $<2 \times$ the upper limit of normal), and renal function (creatinine $<1.5 \mathrm{mg} / \mathrm{dL}$ or calculated creatinine clearance $\geq 60 \mathrm{~mL} / \mathrm{min}$ ) before starting therapy. All patients signed informed consent forms approved by the University of California, San Francisco, Committee on Human Research.

\section{Treatment Plan}

Radiotherapy was administered in doses of 1.8 to 2.0 Gy/d delivered 5 days per week to a total dose of 59.4 to 60 Gy given over a 6-week course. During radiotherapy, patients not receiving enzyme-inducing antiepileptic drugs (EIAEDs; group A) received erlotinib at $100 \mathrm{mg} / \mathrm{d}$ on a continuous basis 7 days/wk. Those receiving EIAEDs (group B) received erlotinib at $200 \mathrm{mg} / \mathrm{d}$. All patients were given temozolomide at $75 \mathrm{mg} / \mathrm{m}^{2} / \mathrm{d}$ continuously 7 days $/ \mathrm{wk}$.

Two weeks after the completion of radiotherapy, patients were treated with temozolomide $200 \mathrm{mg} / \mathrm{m}^{2} / \mathrm{d}$ for 5 days every 28 days. Group A patients received erlotinib at a continuous daily dose of $150 \mathrm{mg} / \mathrm{d}$; group B patients began erlotinib at a dose of $300 \mathrm{mg} / \mathrm{d}$. Dose escalation of erlotinib by $50 \mathrm{mg} / \mathrm{d}$ was allowed every 2 weeks until the development of an intolerable grade 2 or worse rash, up to a maximum dose of $200 \mathrm{mg} / \mathrm{d}$ for group A or $500 \mathrm{mg} / \mathrm{d}$ for group B. Dose escalations for erlotinib were not allowed if the patient developed grade 3 or worse nonhematologic toxicity. Hematologic and liver toxicities were used as criteria for downward adjustment of the temozolomide dose; dose escalation beyond $200 \mathrm{mg} / \mathrm{m} 2 / \mathrm{d}$ was not allowed. Patients were proactively treated to limit diarrhea and skin rash in an attempt to allow dose escalation of erlotinib to at least a grade 2 tolerable rash.

The pretreatment evaluation included a complete history, physical, and neurologic examination. Prestudy laboratory tests, obtained within 14 days of treatment, included a $\mathrm{CBC}$ count with differential, serum creatinine, total bilirubin, AST, ALT, alkaline phosphatase, blood urea nitrogen, glucose, potassium, sodium, anticonvulsant level (if applicable), and serum pregnancy test for women of childbearing potential. Pathology slides from the most recent surgical material were submitted for retrospective pathology review to confirm the diagnosis, as well as to evaluate molecular abnormalities in the tumor, including EGFR by immunohistochemistry (IHC) and fluorescent in situ hybridization, EGFR vIII by IHC, PTEN by IHC, and promoter methylation of the methylguanine methyltransferase (MGMT) gene by a methylation-specific polymerase chain reaction-based assay. ${ }^{9}$ The IHC assays were scored using a 0 to +3 scoring system. No positive staining was scored 0 ; at least $25 \%$ immunoreactivity of cells was scored $+1 ; 26 \%$ to $75 \%$ was scored +2 ; and $76 \%$ or greater was scored +3 . Analyses of results were done using the actual IHC score, and the level of positivity was included as part of the assessment. Thus any level of positivity was considered positive, but the range was taken into account.

During radiation, a $\mathrm{CBC}$ count and differential were performed every 2 weeks. After radiation, $\mathrm{CBC}$ count and differential were performed at weeks 3 and 4 after the start of each 28-day temozolomide cycle. Creatinine, blood urea nitrogen, total bilirubin, AST, ALT, alkaline phosphatase, sodium, and potassium measurements were performed every 8 weeks. Anticonvulsant blood levels of patients on EIAEDs were measured every 8 weeks. Brain magnetic resonance imaging was performed as a baseline (within 14 days before the start of treatment), 2 weeks after the completion of radiation, and then every 8 weeks while patients were receiving treatment.

The intent was to treat up to 12 months; additional treatment beyond 12 months was allowed at the discretion of the treating physician, assuming no significant toxicity, and with the consent of the patient. All patients were observed for overall survival. Patients who experienced disease progression were observed for survival every 3 months.

\section{Evaluation of Response}

The primary end point was overall survival, and time to progression served as a secondary end point. The following imaging guidelines were used to evaluate progression: (1) $25 \%$ increase in the sum of products of all measurable lesions over smallest sum observed (over baseline if no decrease) using the same techniques as baseline, (2) clear worsening of any assessable disease, (3) appearance of any new lesion/site, or (4) clear clinical worsening or failure to return for evaluation as a result of death or deteriorating condition (unless clearly unrelated to this cancer). Progression-free survival (PFS) was defined from the date of diagnosis to the date progressive disease was first observed, nonreversible neurologic progression or permanently increased corticosteroid requirement, death from any cause, or early discontinuation of treatment. Overall survival was defined from the date of diagnosis to date of death from any cause.

\section{Statistical Plan}

The primary end point was survival measured from diagnosis. For the final analysis, results were compared with results of comparable patients treated at University of California, San Francisco, on two previous prospective phase II trials. The first study included the use of thalidomide in combination with temozolomide during and after radiotherapy; the second study included the use of cis-retinoic acid with temozolomide during and after radiotherapy. ${ }^{10,11}$ The primary analysis used a Cox proportional hazards model that included age, KPS, and extent of resection. Initial calculation of sample size was based on a binary end point, with the goal of increasing survival at the historical median survival time ( 14 months) from $50 \%$ to $67 \%$. Sixty patients provided $90 \%$ power using a one-sided $\alpha$ of 0.1 for this binary comparison. This represents a hazard ratio of 0.58 (experimental/historical). An increase in 14 -month survival to $63 \%$, corresponding to a hazard ratio of 0.67 , was also of interest.

Enrollment was expected to occur over a 12- to 15-month period. Follow-up for all patients was to be at least 14 months. A $10 \%$ increase in accrual was allowed to ensure an adequate sample size if any adjustment for ineligible patients was needed. Analysis of biologic correlative data was performed to increase our knowledge of how the potential benefit of erlotinib (if present) may be influenced by genetic characteristics of the tumor. This was an exploratory aim for this study.

A stopping rule was used for the study. If the discontinuation rate owing to toxicity was $\geq 20 \%$ and the lower bound for the one-tailed $95 \%$ CI was more than $10 \%$, the treatment strategy was not considered feasible, and the study would have been discontinued. 


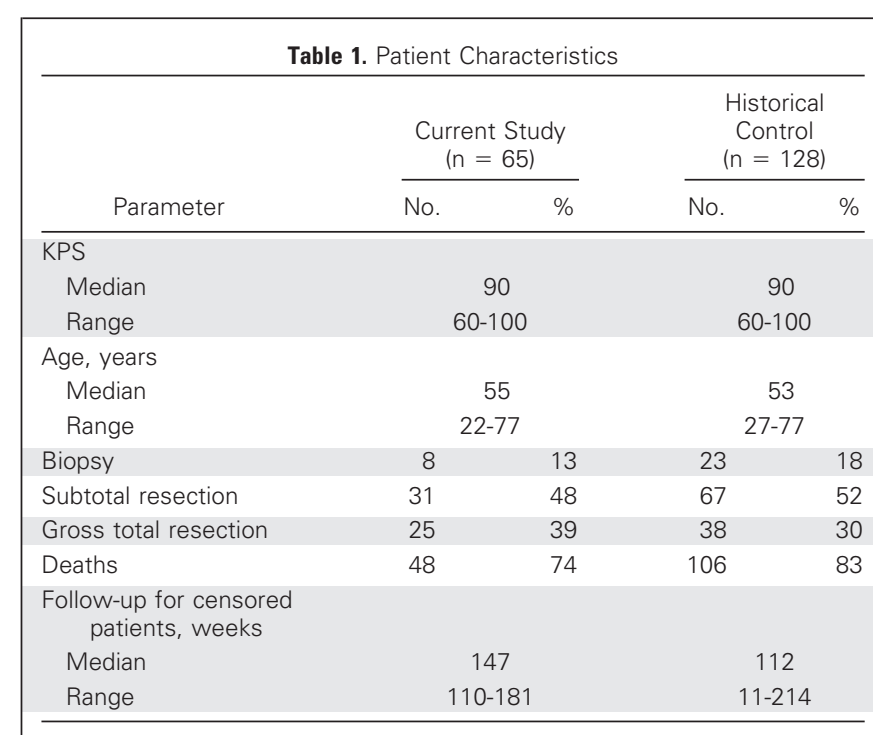

Abbreviation: KPS, Karnofsky Performance Score.

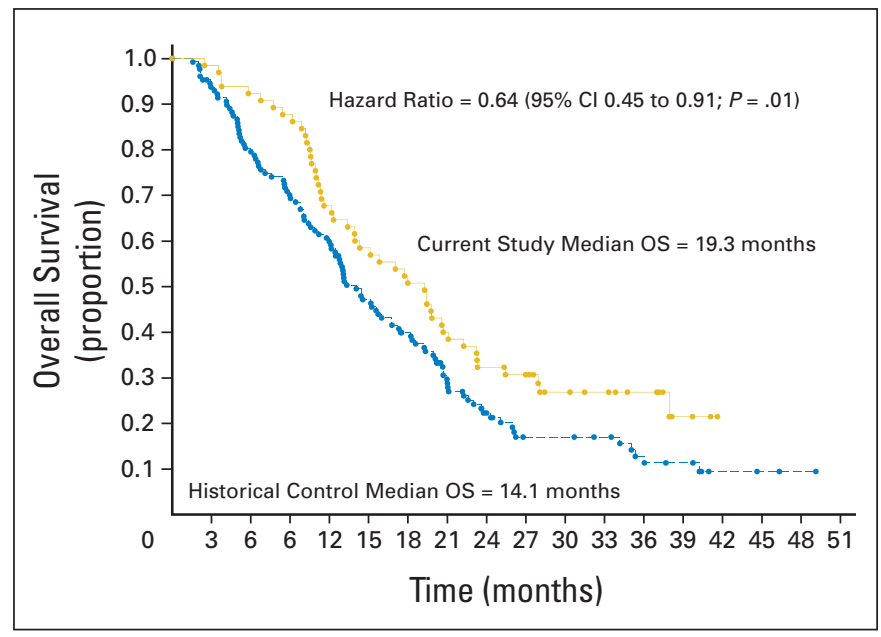

Fig 2. Survival by Kaplan-Meier analysis, comparing the current study with erlotinib plus temozolomide to the historical control studies. The hazard ratio is corrected for age, Karnofsky Performance Score, and extent of resection. Hazard ratio $=0.64(95 \% \mathrm{Cl}, 0.45$ to $0.91 ; P=.01)$. OS, overall survival.

In this study, median PFS was 8.2 months and median survival was 19.3 months. The corresponding results for PFS and median survival for the historical control group were 4.9 months and 14.1 months, respectively (Figs 1 and 2). The hazard ratio for PFS was 0.56 (95\% CI, 0.40 to $0.79 ; P=.001$ ); the hazard ratio for survival was 0.64 ( $95 \% \mathrm{CI}, 0.45$ to $0.91 ; P=.01$ ). The hazard ratio was corrected for age, KPS, and extent of surgery. The 14-month survival rate was $60 \%$. Molecular studies were available in a subset of patients. Not all patients had available paraffin blocks, and in some cases, there was not enough tissue remaining to accomplish each of the correlative studies. Table 2 describes the results of the IHC, fluorescence in situ hybridization, and methylation-specific polymerase chain reaction assays. After controlling for patient factors, promoter methylation of MGMT had the

\begin{tabular}{|c|c|c|c|c|}
\hline Marker & $\begin{array}{c}\text { No. of } \\
\text { Patients }\end{array}$ & Value (Score) & $\begin{array}{c}\text { No. of } \\
\text { Patients }\end{array}$ & $\%$ \\
\hline \multirow[t]{4}{*}{ EGFR by IHC } & \multirow[t]{4}{*}{63} & 0 & 17 & 27 \\
\hline & & +1 & 3 & 5 \\
\hline & & +2 & 9 & 14 \\
\hline & & +3 & 34 & 54 \\
\hline \multirow[t]{4}{*}{ EGFR vIII by IHC } & \multirow[t]{4}{*}{58} & 0 & 52 & 90 \\
\hline & & +1 & 2 & 3 \\
\hline & & +2 & 2 & 3 \\
\hline & & +3 & 2 & 3 \\
\hline \multirow[t]{2}{*}{ EGFR by FISH } & \multirow[t]{2}{*}{51} & Yes (amplified) & 22 & 43 \\
\hline & & No (not amplified) & 29 & 57 \\
\hline \multirow[t]{4}{*}{ PTEN by IHC } & \multirow[t]{4}{*}{52} & 0 & 11 & 21 \\
\hline & & +1 & 21 & 40 \\
\hline & & +2 & 10 & 19 \\
\hline & & +3 & 10 & 19 \\
\hline \multirow{2}{*}{$\begin{array}{l}\text { MGMT promoter } \\
\text { methylation } \\
\text { by MS-PCR }\end{array}$} & \multirow[t]{2}{*}{44} & Yes (methylated) & 16 & 36 \\
\hline & & No (unmethylated) & 28 & 64 \\
\hline \multicolumn{5}{|c|}{$\begin{array}{l}\text { Abbreviations: EGFR, epidermal growth factor receptor; IHC, immunohisto- } \\
\text { chemistry; FISH, fluorescent in situ hybridization; PTEN, phosphatase ano } \\
\text { tensin homolog gene; MGMT, methylguanine methyltransferase; MS-PCR } \\
\text { methylation-specific polymerase chain reaction. }\end{array}$} \\
\hline
\end{tabular}


strongest correlation with survival. The median survival of the 16 patients with MGMT promoter methylation was 25.5 months, and the 2 -year survival rate was $56 \%$. The median survival of the 28 patients without promoter-methylated MGMT was 14.6 months, and the 2-year survival rate was $14 \%$ (hazard ratio $=0.31 ; 95 \% \mathrm{CI}, 0.14$ to $0.71 ; P=.006)$. These data are comparable to a retrospective subset analysis of a larger historical phase III study conducted by the European Organization for Research and Treatment of Cancer and National Cancer Institute of Cancer that randomly assigned patients to receive radiation alone or radiation plus temozolomide, showing a median and 2-year survival rate of 21.7 months and $46 \%$, respectively (Table 3). In the proportional hazards model of data from the current study that included EGFR overexpression, PTEN positivity shown by IHC, and MGMT promoter methylation, there was a statistical interaction between methylation status and PTEN positivity $(P=.01)$. Given this strong interaction, a proportional hazards model correcting for KPS, age, and extent of resection was evaluated that included MGMT promoter methylation status, PTEN by IHC, and EGFR by IHC. Although the numbers are small, there seems to be a survival benefit for patients who are both MGMT promoter methylated and PTEN positive (hazard ratio $=0.20$ comparing those PTEN positive $v$ negative among the MGMT promoter-methylated patients). The overall model was not as robust for patients without promoter methylation, but a potential trend toward improved survival for nonmethylated patients who were EGFR overexpressing was noted (hazard ratio, 0.65). Tables 3 and 4 describe the molecular correlation of biomarkers and survival. Because of the small number of patients with the other molecular markers or combinations of markers, additional analyses were not performed.

Toxicity was modest and tolerable (Table 5). The majority of treatment-specific adverse events were grade 3 , and there were no grade 5 events related to treatment. Hematologic and liver function abnormalities were considered to be most likely related to temozolomide, rash and diarrhea were related to erlotinib, and fatigue was related to both drugs, as well as to radiotherapy. The majority of patients (22 of 28) not receiving EIAEDs were adjuvantly treated with at least a $150 \mathrm{mg} / \mathrm{d}$ dose of erlotinib. Of the 37 patients receiving EIAEDs, 27 patients were treated with $300 \mathrm{mg}$ or less, and the remaining 10 patients were treated to a dose ranging from 350 to $500 \mathrm{mg} / \mathrm{d}$. Reasons for an inability to reach a higher dose included a need to

\begin{tabular}{|lcl|}
\hline \multicolumn{2}{|c|}{ Table 3. MGMT Methylation Status and Outcome } \\
\hline Methylation Status & Current Study* & $\begin{array}{c}\text { EORTC Phase } \\
\text { III Trialt }\end{array}$ \\
\hline MGMT promoter methylated & 46 \\
No. of patients & 16 & 21.7 \\
Median survival, months & 25.5 & 46 \\
2-year survival, \% & 56 & 60 \\
MGMT not promoter methylated & 28 & 12.7 \\
No. of patients & 14.6 & 13.8 \\
Median survival, months & 14 & \\
2-year survival, \% & \\
\hline Abbreviations: MGMT, methylguanine methyltransferase; EORTC, European \\
Organization for Research and Treatment of Cancer. \\
*Hazard ratio = 0.31 (95\% Cl, 0.14 to 0.71; $P=.006)$. \\
†Subset analysis of patient survival from phase III trial. ${ }^{12}$ & \\
\hline
\end{tabular}

\begin{tabular}{|c|c|c|}
\hline \multicolumn{3}{|c|}{$\begin{array}{l}\text { Table 4. Molecular Correlations Using Proportional Hazards Adjusting } \\
\text { for Age, Karnofsky Performance Score, and Extent of Resection* }\end{array}$} \\
\hline Variable & Hazard Ratio & $P$ \\
\hline \multicolumn{3}{|c|}{$\begin{array}{l}\text { MGMT promoter methylated, } \mathrm{n}=14 \dagger \\
\quad(P \text { for overall model }=.05)\end{array}$} \\
\hline PTEN by IHC, 0, 1, 2, 3 & 0.20 & .04 \\
\hline EGFR by $I H C, 0,1,2,3$ & 0.82 & .68 \\
\hline \multicolumn{3}{|c|}{$\begin{array}{l}\text { MGMT unmethylated, } \mathrm{n}=23+ \\
\quad(P \text { for overall model }=.15)\end{array}$} \\
\hline PTEN by IHC, 0, 1, 2, 3 & 1.32 & .22 \\
\hline EGFR by IHC, 0, 1, 2, 3 & 0.65 & .06 \\
\hline \multicolumn{3}{|c|}{$\begin{array}{l}\text { Abbreviations: MGMT, methylguanine methyltransferase; PTEN, phospha- } \\
\text { tase and tensin homolog gene; IHC, immunohistochemistry; EGFR, epidermal } \\
\text { growth factor receptor. } \\
\text { "*In the model that included EGFR by IHC, PTEN by IHC, and MGMT promoter } \\
\text { methylation there was a statistical interaction between MGMT promoter } \\
\text { methylation status and PTEN ( } P=.01 \text { ). } \\
\text { TSample size is reduced because of missing values for one or both of EGFR } \\
\text { and PTEN. }\end{array}$} \\
\hline
\end{tabular}

change from an EIAED to a non-EIAED, early tumor progression, or the development of intolerable grade 2 rash.

\section{DISCUSSION}

The current standard of care for treating patients with newly diagnosed GBM is based on a randomized phase III trial published in $2005 .^{2}$ In that study, patients were treated either with radiation alone or radiation with concurrent temozolomide followed by adjuvant temozolomide given for 6 months. Median survival for the temozolomide-plus-radiotherapy arm was 14.6 months as compared with 12.1 months for the radiotherapy-only arm. The 2-year survival rate for the temozolomide-treated group was $26 \%$, compared with $10 \%$ for the radiation-only group. A posthoc subgroup analysis of patients treated in this trial correlating MGMT promoter methylation with survival was conducted in an attempt to define patient groups that may be more or less sensitive to treatment. ${ }^{12}$ Patients with MGMT promoter methylation had significantly improved median survival compared with patients with intact MGMT (21.7 months $v$ 15.3 months).

As encouraging as these results are, however, most patients die of tumor progression. In this study we attempted to improve survival

\begin{tabular}{|lc|}
\hline \multicolumn{2}{|c|}{ Table 5. Treatment-Specific Adverse Events } \\
\hline Type of Adverse Event & No. of Events \\
\hline Grade 3 & 28 \\
Lymphopenia & 6 \\
Liver function abnormality & 4 \\
Fatigue & 1 \\
Diarrhea & 4 \\
Rash & 2 \\
Grade 4 & 2 \\
Neutropenia & 1 \\
Thrombocytopenia & 1 \\
Fatigue & 1 \\
Liver function abnormality & \\
\hline
\end{tabular}


outcome over that seen with temozolomide and radiotherapy. Combining temozolomide with erlotinib and radiotherapy did reveal encouraging results. Compared with the historical phase II studies conducted at our institution, this new trial seems to show superior outcomes. $^{10,11}$ We chose the two historical studies for comparator trials based on the similarity of the three trials in terms of patient characteristics, eligibility requirements, and imaging standards, as well as the use of temozolomide during and after radiotherapy. In addition, both historical studies added a noncytotoxic agent to radiation and temozolomide. The previous studies did not represent a significant improvement over that seen with radiotherapy and temozolomide alone. Of particular interest with the current study is the improvement in both progression-free and median survival. It should be noted that few patients were declared to have experienced disease progression at the initial postradiation assessment, well beyond the time frame when pseudoprogression may have occurred. We feel encouraged that the longer PFS in this study was due to a positive and sustained treatment effect.

The molecular correlative studies in this trial confirm the survival advantage seen in patients with MGMT promoter methylation and also suggest an interaction between MGMT and PTEN status. One significant observation was the inability to escalate the dose of erlotinib to our planned higher dose in patients treated with EIAEDs. It is possible that erlotinib exposure was reduced in this subset of patients. Whether higher doses or improved systemic exposure would have further increased survival in these patients is unknown, however, as pharmacokinetic data were not collected from these patients, the patient subset was small, and other patient factors may have influenced outcome. Our results must be viewed with the caution that this was a small, phase II study conducted in a single institution.

We and others have tested several agents in combination with temozolomide, using agents that showed in vitro and in vivo impact on specific molecular targets that seem relevant to glioma cell growth. Targeting EGFR is a strategy based both on preclinical and preliminary clinical human data in patients with recurrent malignant glioma. Phase I testing of the combination of temozolomide and erlotinib documented tolerability, and preliminary evidence of efficacy of single-agent erlotinib, although modest at best, was encouraging. ${ }^{7}$ Studies of the combination of temozolomide and the EGFR inhibitor gefitinib (Iressa; AstraZeneca, Wilmington, DE) have also been conducted, demonstrating tolerability and evidence of efficacy in recurrent disease. ${ }^{13}$ However, phase I and II studies of gefitinib and erlotinib used as single agents adjuvant to radiotherapy have shown no major improvements in survival for patients. ${ }^{14-16}$ None of these studies included temozolomide in the treatment regimen, and it seems that single-agent treatment with an EGFR inhibitor in newly diagnosed disease may not be beneficial.

There is presumptive evidence that molecular profiles may predict response to EGFR inhibitors in at least some subgroups of patients. ${ }^{8,17}$ In previous studies, patients with recurrent disease were more likely to respond to erlotinib or gefitinib if their tumors were found to be either EGFR overexpressed, amplified, or mutated, plus intact for PTEN, or without Akt phosphorylation. Other studies, however, have not shown such correlations. ${ }^{18}$ In this current study, we found a significant difference in outcome, both for median and 2-year survival expectations, between patients with promoter-methylated MGMT and those with unmethylated MGMT. This result is consistent with the subgroup analysis performed by the European Organization for Research and Treatment of Cancer/National Cancer Institute of Canada phase III trial. ${ }^{12}$ In addition, patients who had MGMT gene silencing and intact PTEN had a significant survival advantage. Although this is not direct evidence, it at least suggests that some patient groups may be influenced and possibly identified by this specific molecular signature. The MGMT promoter methylation status was not evaluated in our previous two clinical trials, and we cannot directly compare survival outcomes within this molecular context. Attempts are ongoing to retrieve tumor blocks from those patients. However, there is no reason to believe that the general mix of tumor types will have changed substantially over this interval, and the absence of this information must be considered one of the random factors that need to be recognized in any clinical trial comparison using historical data sets. These observations need much more testing, particularly in larger, prospective, controlled clinical studies, and currently should not be used to stratify patients.

In conclusion, the current study shows encouraging PFS and median survival over that seen in our previous studies. We feel that further evaluation of this combination is warranted in a larger prospective trial, with additional molecular correlative studies.

\section{AUTHORS' DISCLOSURES OF POTENTIAL CONFLICTS OF INTEREST}

Although all authors completed the disclosure declaration, the following author $(s)$ indicated a financial or other interest that is relevant to the subject matter under consideration in this article. Certain relationships marked with a " $U$ " are those for which no compensation was received; those relationships marked with a " $C$ " were compensated. For a detailed description of the disclosure categories, or for more information about ASCO's conflict of interest policy, please refer to the Author Disclosure Declaration and the Disclosures of Potential Conflicts of Interest section in Information for Contributors.

Employment or Leadership Position: David Stokoe, Genentech (C) Consultant or Advisory Role: Susan M. Chang, Genentech (C) Stock Ownership: David Stokoe, Genentech Honoraria: None Research Funding: Michael D. Prados, Genentech; Susan M. Chang, Schering Plough Expert Testimony: None Other Remuneration: None

\section{AUTHOR CONTRIBUTIONS}

Conception and design: Michael D. Prados, Susan M. Chang, Kathleen R. Lamborn, Daphne A. Haas-Kogan

Financial support: Michael D. Prados

Administrative support: Rebecca DeBoer, Rupa Parvataneni, Hannah Carliner, Jane Rabbitt, Margaretta Page, Anne Fedoroff

Provision of study materials or patients: Michael D. Prados, Susan M. Chang, Nicholas Butowski, Penny K. Sneed, Mitchel S. Berger, Michael W. McDermott, Andrew T. Parsa

Collection and assembly of data: Michael D. Prados, Rebecca DeBoer, Rupa Parvataneni, Hannah Carliner, Paul Kabuubi, Jennifer Ayers-Ringler, Jane Rabbitt, Margaretta Page, Anne Fedoroff, Scott Vandenberg, C. David James, Kathleen R. Lamborn, David Stokoe Data analysis and interpretation: Michael D. Prados, Scott Vandenberg, C. David James, Kathleen R. Lamborn, David Stokoe, Daphne A. Haas-Kogan

Manuscript writing: Michael D. Prados, Susan M. Chang, Rebecca DeBoer, Rupa Parvataneni, Kathleen R. Lamborn, Daphne A. Haas-Kogan

Final approval of manuscript: Michael D. Prados, Penny K. Sneed, Scott Vandenberg, Kathleen R. Lamborn, Daphne A. Haas-Kogan 


\section{REFERENCES}

1. Central Brain Tumor Registry of the United States Statistical Report: Primary Brain Tumors in the United States, 1997-2001. Chicago, IL, Central Brain Tumor Registry of the United States, 2004

2. Stupp R, Mason WP, van den Bent MJ, et al: Radiotherapy plus concomitant and adjuvant temozolomide for glioblastoma. N Engl J Med 352:987996, 2005

3. Ekstrand AJ, Sugawa $N$, James $C D$, et al: Amplified and rearranged epidermal growth factor receptor genes in human glioblastomas reveal deletions of sequences encoding portions of the $\mathrm{N}$ and/or C-terminal tails. Proc Natl Acad Sci U S A 89:4309-4313, 1992

4. Frederick L, Wang XY, Eley G, et al: Diversity and frequency of epidermal growth factor receptor mutations in human glioblastomas. Cancer Res 60: 1383-1387, 2000

5. Feldkamp MM, Lala P, Lau N, et al: Expression of activated epidermal growth factor receptors, Ras-guanosine triphosphate, and mitogen-activated protein kinase in human glioblastoma multiforme specimens. Neurosurgery 45:1442-1453, 1999

6. Fry DW: Inhibition of the epidermal growth factor receptor family of tyrosine kinases as an approach to cancer chemotherapy: Progression from reversible to irreversible inhibitors. Pharmacol Ther 82:207-218, 1999

7. Prados MD, Lamborn KR, Chang $S$, et al: Phase 1 study of erlotinib $\mathrm{HCl}$ alone and combined with temozolomide in patients with stable or recurrent malignant glioma. Neuro Oncol 8:67-78, 2006

8. Haas-Kogan DA, Prados MD, Tihan $T$, et al: Epidermal growth factor receptor, protein kinase B/Akt, and glioma response to erlotinib. J Natl Cancer Inst 97:880-887, 2005

9. Esteller $\mathrm{M}$, Hamilton SR, Burger $\mathrm{PC}$, et al: Inactivation of the DNA repair gene 06-methylguanineDNA methyltransferase by promoter hypermethylation is a common event in primary human neoplasia. Cancer Res 59:793-797, 1999

10. Chang SM, Lamborn KR, Malec M, et al: Phase II study of temozolomide and thalidomide with radiation therapy for newly diagnosed glioblastoma multiforme. Int J Radiat Oncol Biol Phys 60: 353-357, 2004

11. Butowski N, Prados MD, Lamborn KR, et al: A phase II study of concurrent temozolomide and cis-retinoic acid with radiation for adult patients with newly diagnosed supratentorial glioblastoma. Int J Radiat Oncol Biol Phys 61:1454-1459, 2005

12. Hegi ME, Diserens AC, Gorlia T, et al: MGMT gene silencing and benefit from temozolomide in glioblastoma. N Engl J Med 352:997-1003, 2005
13. Prados MD, Yung WK, Wen PY, et al: Phase-1 trial of gefitinib and temozolomide in patients with malignant glioma: A North American brain tumor consortium study. Cancer Chemother Pharmacol 61:1059-1067, 2008

14. Uhm JH, Ballman KV, Giannini $C$, et al: Phase II study of ZD1839 in patients with newly diagnosed grade 4 astrocytoma. J Clin Oncol 22:14s, 2004 (suppl; abstr 1505)

15. Chakravarti $A B$, Robins HI, Guha $A$, et al: An update of phase II results from RTOG 0211: A phase I/II study of gefitinib with radiotherapy in newly diagnosed glioblastoma. J Clin Oncol 24:18s, 2006 (suppl; abstr 1527)

16. Krishnan S, Brown PD, Ballman KV, et al: Phase I trial of erlotinib with radiation therapy in patients with glioblastoma multiforme: Results of North Central Cancer Treatment Group protocol N0177. Int J Radiat Oncol Biol Phys 65:1192-1199, 2006

17. Mellinghoff IK, Wang MY, Vivanco I, et al: Molecular determinants of the response of glioblastomas to EGFR kinase inhibitors. N Engl J Med 353:2012-2024, 2005

18. Lassman $A B$, Rossi MR, Raizer $\mathrm{JJ}$, et al: Molecular study of malignant gliomas treated with epidermal growth factor receptor inhibitors: Tissue analysis from North American Brain Tumor Consortium Trials 01-03 and 00-01. Clin Cancer Res 11: 7841-7850, 2005

\section{Acknowledgment}

We thank Ilona Garner, Department of Neurological Surgery, University of California, San Francisco, for editorial support. 